\title{
Choosing Between the Two-sample $T$ Test and its Alternatives: A Practical Guideline
}

\author{
Julian D. Karch ${ }^{1}$ \\ ${ }^{1}$ University
}

\begin{abstract}
The traditional guideline for choosing between the two-sample $t$ test and its alternatives is primarily based on assessing assumptions. Many flaws of this approach have been documented. In this paper, I address those flaws briefly and propose a new guideline for choosing between the two-sample $t$ test and its alternatives. I propose to select the hypothesis that operationalizes the research question best. This selection is carried out before data collection and entails identifying the hypotheses that in principle produce meaningful results, and among those, the most appropriate one. For testing the selected hypotheses, I recommend bootstrap and permutation tests instead of the traditionally used parametric tests. The role of assessing assumptions is downgraded to deciding whether the results of a test are reliable. An important implication of the proposed guideline is that a nonparametric permutation test should be used instead of the $t$ test in most cases. While this new guideline focuses on hypothesis testing, large parts are also relevant for choosing a confidence interval method due to the close correspondence between confidence intervals and hypothesis tests.
\end{abstract}

Keywords: t test, choosing, practical guideline, permutation, hypothesis testing, nonparametric

\section{Introducti}

For answering many psychological research questions, two independent groups are compared. Typically, it is desired to establish whether in one group the values of a variable of interest tend to be higher, for example, to verify that a new therapy method leads to better outcomes. In line with Schlag (2015), I call this goal establishing direction.

The standard approaches for establishing direction between two independent groups are the two-sample $t$ test and the Wilcoxon-Mann-Whitney test ${ }^{1}$ and their associated confidence intervals. The traditional guideline for choosing between these tests (see, for example, Field, 2017, Chapter 6) relies on the principle of using the two-sample $t$ test as default and switching to the Wilcoxon-Mann-Whitney test only if the $\mathrm{t}$ test's assumptions seem so heavily violated that the results are considered invalid.

Two assumptions of the recommended (Delacre et al., 2017; Ruxton, 2006) Welch version ${ }^{2}$ of the $t$ test are nonnormal data and no outliers (Field, 2017). As the first step, each assump-

Correspondence concerning this article should be addressed to Julian D. Karch, Methodology and Statistics Department, Institute of Psychology, Leiden University, PO Box 9555, 2300 RB Leiden. E-mail: j.d.karch@fsw.leidenuniv.nl tion is assessed based on the observed data. For normality, techniques that assess how strong normality is violated are employed, for example, a quantile-quantile plot (Field, 2017). The most common approach for assessing outliers relies on z-scores (Bakker \& Wicherts, 2014). In an optional second step, it is attempted to alleviate identified problems. For example, transformations are applied in the hope of making the data more normal (Field, 2017). Alternatively, moderate nonnormality is and can often be ignored when the sample size is large enough due to the type I error robustness of the $t$ test to this assumption (Fay \& Proschan, 2010). Outliers are often removed with the hope of safeguarding the validity of the $t$ test (André, 2021; Bakker \& Wicherts, 2014). Only if the problems in the data are deemed severe enough to invalidate the $t$ test's results and cannot be corrected is the WilcoxonMann-Whitney test used (Field, 2017).

The traditional guideline has been criticized for multiple reasons:

Preliminary testing paradox: Instead of maintaining the whole procedure's validity, choosing between the Wilcoxon-MannWhitney and the $t$ test based on preliminary tests of assumptions distorts the type I and II error rates (Rasch et al., 2011).

\footnotetext{
${ }^{1}$ Alternatively known as Mann-Whitney $U$, Mann-Whitney-Wilcoxon, or Wilcoxon rank-sum test

${ }^{2}$ This test is also known as unequal variances $t$ test and thus, in contrast to Student's $t$ test, does not assume equal variances.
} 
Outlier removal paradox: Instead of maintaining the validity of the $t$ test, removing outliers leads to inflated type I error rates and invalid confidence intervals (André, 2021; Bakker \& Wicherts, 2014).

Observed data completely determine research question: By changing from the $t$ test to the Wilcoxon-Mann-Whitney based on assessing assumptions, the hypothesis is determined only by characteristics of the data and not by the research question (Delaney \& Vargha, 2002; Field, 2017, sec. 6.12.5).

Measurement issues are neglected: For example, it is often not meaningful to compare means if the variable is not at least interval scaled (Cliff, 1996a).

Modern methods are ignored: Modern methods, such as robust tests (Wilcox, 2016), resampling tests (Good, 2005), and recent improvements over the Wilcoxon-Mann-Whitney test (Brunner et al., 2019; Brunner \& Munzel, 2000; Cliff, 1996b; Neubert \& Brunner, 2007) are ignored.

Multiple new practical guidelines for choosing between the two tests and their variants have been proposed (Cliff, 1996b; Rietveld \& van Hout, 2015; Ruxton \& Neuhäuser, 2019). While these new guidelines are improvements, none addresses all the criticism. Exemplary, Ruxton and Neuhäuser (2019), neglect measurement issues, and Rietveld and van Hout (2015) let the characteristics of the observed data completely determine the research question.

In this paper, I propose a new practical guideline for choosing between the two tests and their frequentist variants addressing all the criticism. The guideline focuses on selecting between hypothesis tests. However, since each considered hypothesis test has an associated confidence interval estimator, it is also valuable for selecting a confidence interval estimator. The resulting guideline should not be considered optimal ${ }^{3}$, and I explicitly invite criticism to improve it. However, I am convinced that it is a substantial improvement over the existing guidelines and should thus be employed instead.

In the body of this paper, I will describe the guideline and its rationale, as well as demonstrate it on an example data set. In the discussion, I will show that it addresses all the criticism. In the supplementary material (https://osf.io/2e6jf/), I address practicalities such as how to perform and report on the recommended hypotheses tests.

\section{Step One: Identify Most Appropriate Hypothesis}

\section{Example Data Set}

Alongside the description of the new guideline, I will demonstrate it by applying it to an example data set. The data originate from the 1994 wave of the Canadian Survey of Labour and Income Dynamics for the province of Ontario
(Fox, 2016) and are available as the SLID data set within the carData $\mathrm{R}$ package (Fox et al., 2020). Among other variables, the SLID dataset contains the participants' wage, quantified as composite hourly wage rate from all jobs. The research question I exemplarily investigate is whether one of the two sexes earns more.

\section{Overview}

The first step towards the new guideline is realizing that the tests investigate profoundly different null and alternative hypotheses and are thus appropriate for different research questions. The null hypothesis of the $t$ test is equality of means, and when testing two-sided ${ }^{4}$ the alternative hypothesis is inequality of means. The associated population parameter is the difference in means. For the Wilcoxon-Mann-Whitney test, the null hypothesis is stochastic equality (Divine et al., 2018) ${ }^{5}$ the alternative hypothesis stochastic inequality. The associated population parameter is stochastic superiority, which is the probability that a random observation from one group is bigger than a random observation from the other, with ties split evenly. Two groups are stochastically equal if and only if the stochastic superiority is $50 \%$. Those different hypotheses can lead to different and even opposite conclusions since, for example, two groups can have the same mean but be stochastically unequal. Within the context of the worked example, the $t$ test thus considers males and females to earn the same if their average hourly wage rate is the same. In contrast, the Wilcoxon-Mann-Whitney test concludes this if the probability of a random female earning more than a random male is $50 \%$.

This realization leads to the first step of the new guideline. Identify the hypothesis ${ }^{6}$ that is most appropriate for the research question. Thus, the hypothesis is selected before data collection and independently of the observed data's characteristics. This is in line with the straightforward argument that one should test what one wants to know (Delaney \& Vargha, 2002; Wilcox, 2017, p. 391). However, it conflicts with the

\footnotetext{
${ }^{3}$ As will become clear later, an optimal guideline is out of reach at the moment.

${ }^{4}$ When testing one-sided, the alternative hypothesis is that one mean is bigger. I will focus on two-sided testing in this paper and will not discuss how to choose between one-sided and two-sided testing, as the traditional approach (see, e.g., Field, 2017) for this is valid.

${ }^{5}$ Under certain assumptions, the Wilcoxon-Mann-Whitney test is also valid for different hypotheses (Fay \& Proschan, 2010). However, it has been it has repeatedly been argued to be the most appropriate hypothesis for the Mann-Whitney test is stochastic equality (Chung \& Romano, 2013; Divine et al., 2018).

${ }^{6}$ With hypothesis, I refer to the combination of null hypothesis and alternative hypothesis. I will describe such a hypothesis by its null hypothesis. The alternative hypothesis is implied, as it is the opposite of the null hypothesis.
} 
traditional strategy, which selects the hypothesis test and thus the tested hypothesis almost exclusively based on characteristics of the observed data. This causes many problems raised against the traditional strategy, such as the preliminary testing paradox. Consequently, I intentionally avoid it.

For finding the most appropriate hypothesis, a two-step procedure is recommended. First, identify the meaningful hypotheses, which are those that, in principle, produce meaningful results. Second, of the meaningful hypotheses, identify the most appropriate. Importantly, the remaining meaningful hypotheses often provide valuable complementary information and should thus be examined in a secondary analysis. The hypotheses tested by the $t$ test, the WMW test and their variants are comparing means, comparing trimmed means, and assessing stochastic equality, for which the traditional tests are Student's $t$ test, Yuen-Welch's test, and the WilcoxonMann-Whitney test, respectively. An $x \%$ trimmed mean is the mean after discarding the $x \%$ smallest and largest values.

Note that identifying the most appropriate hypothesis is not limited to hypothesis testing but is equally relevant for selecting the most appropriate confidence interval method. For example, if the guideline recommends testing equality of means, this directly implies that a confidence interval for the differences in means should be reported.

Complementary to the textual description, the process of identifying the most appropriate hypothesis is visualized in Figure 1.

\section{Identify Meaningful Hypotheses}

\section{Research Question Allows Treating the Observed Variable as at Least Ordinal?}

The first step is to ensure that the research question allows treating the observed variable as at least ordinal. If this is not the case, direction can not be established, and no hypothesis discussed here is meaningful. The traditional treatment of measurement scales (Stevens, 1946) assigns measurement levels to variables independently of the research question. However, here I adopt the approach of considering the measurement level to also depend on the research question (Velleman \& Wilkinson, 1993).

I will illustrate by adapting an example from the seminal paper of (Lord, 1953). Suppose there is a professor that is responsible for distributing football numbers for the teams of a university. In a departure from reality, only the numbers 1 to 10 are available. Another professor is tasked with overseeing the distribution of numbers; specifically, they are asked to investigate whether 1) none of the teams is favored in the sense that it tends to get higher numbers than the other teams, and 2) the professor does not make any difference between the teams at all, that is, the probabilities for getting each number are the same across the teams. Research question 1) implies an order between the numbers and thus allows treating the numbers as at least ordinal. For research question 2, on the other hand, no order is implied. The answer to that research question is invariant to any recoding of the numbers; they could even be replaced by letters. Thus, research question 1 allows treating the football numbers as ordinal, whereas research question 2 does not.

\section{Research Question Treats Observed Variable as Surrogate for Latent Construct?}

The next question is whether the research question treats the observed variable as a surrogate for an underlying latent construct. I will illustrate using an example. A researcher investigates whether females or males tend to be more depressed. As outcome variable, they obtained the number of depressive episodes in the last year. Here, the number of depressive episodes is not the construct of interest but only a surrogate for the underlying latent construct "severity of depression." If, however, the research question is which sex reports more depressive episodes, then the observed variable is not a surrogate, as it measures the construct of interest directly. Investigating the surrogate question is thus based on the research question and the status of the observed variable.

While I recommend considering all decisions carefully, I introduce heuristic defaults to address the fact that not all researchers will have the time or means to decide on all aspects. For the surrogate question, the recommended heuristic default position is to assume that the observed variable is a surrogate, as this is more common in psychology (Cliff, 1996b) and the weaker assumption.

\section{Linear Relationship Between Observed and Latent Variable?}

If the observed variable is a surrogate, the next question is whether it is reasonable to assume a linear relationship between the latent and the observed variable. This is impossible to assess with absolute certainty, as the latent variable is not observable. In the example, linearity is hard to justify. It would require showing that a person who is twice as severely depressed on average reports twice as many depressive episodes, which does not necessarily seem more plausible than any other monotonic relationship between depressive severity and the number of depressive episodes.

As heuristic default position, not assuming linearity is recommended, as linearity is often implausible in psychology (Cliff, 1996b). 


\section{Figure 1}

Overview of step one: Identify the most appropriate hypothesis.

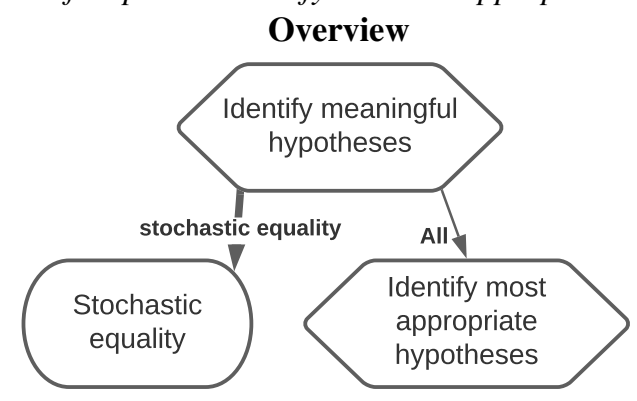

\section{Identify Meaningful Hypotheses}

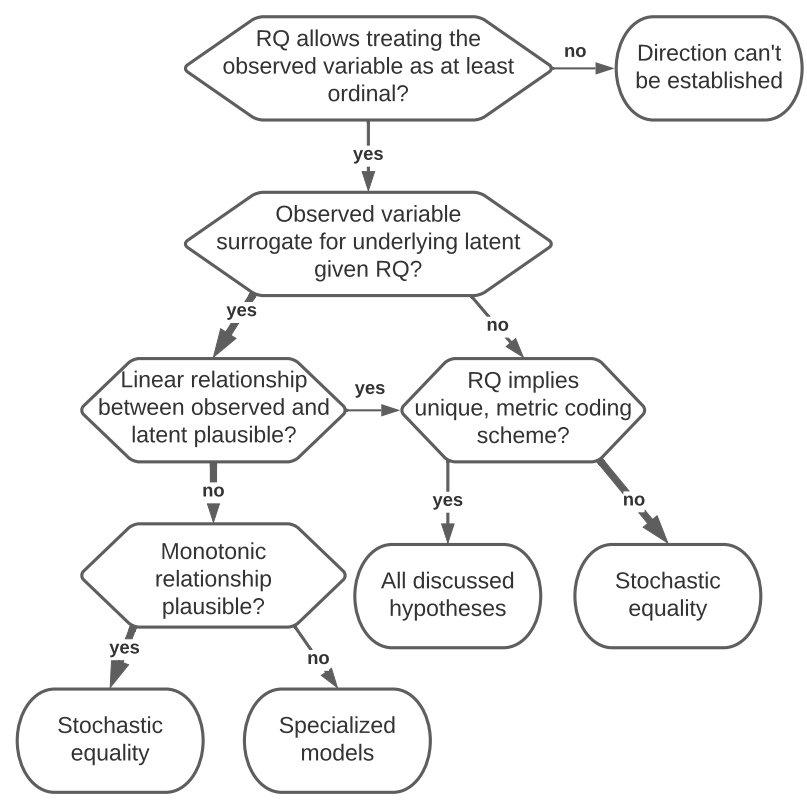

Assign Appropriateness. Indicators for each hypothesis to be most appropriate.

\section{Stochastic Equality}

- Research question concerns direct comparison of individual scores.

\section{Comparison of Trimmed Means}

- Research question concerns comparison of averages

- Most extreme values are not important

\section{Comparison of Means}

- Research question concerns comparison of averages

- Most extreme values are important

Note. Bold lines in the decision tree represent heuristic default choices. $\mathrm{RQ}=$ research question. 
Monotonic Relationship Between Observed and Latent Variable?

In case linearity cannot be justified, one has to establish whether at least monotonicity is plausible, that is, whether larger values of the latent variable lead to larger values of the observed variable (possibly with measurement error). If monotonicity is plausible (but not linearity), the only meaningful hypothesis is stochastic equality (Cliff, 1996b).

Monotonicity is a weaker assumption than linearity and contains linearity as a special case. In the example, this translates into that people who are more depressed tend to report more depressive episodes, which seems plausible.

If monotonicity is not plausible, none of the discussed hypotheses is meaningful, and one has to employ specialized techniques, such as nonlinear latent variable models (Umbach et al., 2017).

\section{Research Question Implies Unique, Metric Coding Scheme?}

If either a linear relationship is reasonable or the observed variable is not a surrogate, one has to establish whether a unique (up to linear transformations), metric coding scheme is implied by the research question. If this is the case, all considered hypotheses are meaningful; if not, only stochastic equality is meaningful (Cliff, 1996b).

Whether a unique metric coding scheme exists is similar to the question of whether a variable is at least interval scaled. However, whether a unique metric coding scheme exists depends not only on the variable but also on the research question (Lord, 1953; Velleman \& Wilkinson, 1993). Essentially, if the research question allows treating the variable as at least an interval variable, then a unique metric coding scheme exists.

I will illustrate using the following example. A researcher wants to validate their new depression therapy. Their research question is whether the new therapy leads to larger improvements than the control treatment. As outcome variable, the depression score after 12 months of therapy is measured as a variable with the levels "no improvement," "slight improvement," "substantial improvement," and "symptom-free." To be able to perform a hypothesis test, numbers need to be assigned to all improvement levels. Under the research question, all coding schemes that encode the ordering are equally valid, for example, 1,2,3,4 and 5,9,15,35. However, the outcome of many hypothesis tests, in particular, comparing (trimmed) means, will differ across coding schemes. In other words, by choosing the right coding scheme, one can manipulate the results such that they are as desired. In contrast, the stochastic equality hypothesis returns the same results for any valid coding scheme and is consequently is the only meaningful hypothesis for this research question.
If, however, the research question is whether applying the therapy leads to an economic benefit for the health care system of a country, the situation is different. To answer this research question, each improvement level must be associated with an (expected) amount of money saved for the health care system. In this case, the only valid metric coding scheme is the amount of money saved, and comparing means is meaningful.

As heuristic default position, assuming that a unique metric coding scheme does not exist is recommended, as it has been repeatedly argued that most often it does not (Cliff, 1996a; Stevens, 1946).

\section{Recurring Example}

Applying the proposed strategy for identifying the meaningful hypotheses to the recurring example leads to the following results. The research question is whether one of the sexes earns more and thus allows treating the hourly wage rate as at least ordinal. The observed variable is not a surrogate for an underlying latent variable since the hourly wage rate directly quantifies how much each person earns. A unique metric coding scheme is implied by the research question, as money earned per hour is a metric variable. Consequently, all hypotheses are meaningful.

\section{Identify Most Appropropriate Hypothesis}

Identifying the meaningful hypotheses can lead to two results: All hypotheses are meaningful, or only stochastic equality is meaningful. If only stochastic equality is meaningful, it should be tested. Otherwise, the most appropriate hypothesis has to be identified.

Which hypothesis is most appropriate generally depends on which hypothesis is the best operationalization of the research question. Consequently, clear decision rules are impossible to establish. Instead, I will list indicators that make it probable that a hypothesis is most appropriate.

If the research question concerns comparing individual scores, then stochastic equality tends to be most appropriate (Delaney \& Vargha, 2002). Consider the following example. A researcher investigates which of two smoking reduction therapies helps the greater number of people. As outcome variable, the reduction in cigarettes smoked per day is available. Assume that for the equal therapy all patients improve slightly, more concretely, by 5 cigarettes per day. For the unequal therapy, $20 \%$ of the patients improve substantially by 20 cigarettes a day, but the remaining $80 \%$ only by 4 cigarettes a day. According to the stochastic equality perspective, the equal therapy should be preferred. When picking two random patients from each group, there is an $80 \%$ probability that the patient following the equal therapy improved more than the patient following the unequal therapy. 
However, stochastic equality is not without weakness and thus not always most appropriate. Specifically, it ignores the magnitude of differences. In the example, the $20 \%$ of patients that profited more from the unequal therapy reduced by 16 cigarettes more than the patients in the equal therapy. In contrast, the $80 \%$ that profited less from the unequal therapy reduced by only 1 cigarette less. A comparison of mean takes these differences in magnitudes into account. Comparing means, the unequal therapy is preferred as it leads to a mean reduction of 7.2 cigarettes a day compared to 5 cigarettes for the equal therapy. Thus, the choice between a comparison of means and stochastic equality should essentially be made by which research question is asked: "for which therapy is the probablity higher that a random person has a higher improvement than the improvement of a random person following the other therapy" or "which therapy leads to better average improvements?".

Comparing trimmed means is similar to comparing means and should thus be preferred in similar situations. The crucial difference is that a comparison of trimmed means ignores a certain proportion of the most extreme values. This has been used to argue that a trimmed mean is a better representation of the average person (Keselman et al., 2004). However, if the most extreme values are crucial, then comparing trimmed means cannot be recommended. When comparing trimmed means, the amount of trimming needs to be chosen carefully, as different amounts of trimming can lead to substantially different results. Optimally, this should be done carefully following, for example, the guidance given in Wilcox (2016). A recommended default value for psychology is $20 \%$ (Wilcox, 2017). For the example, the $20 \%$ trimmed mean perspective leads to the same conclusion as the stochastic equality perspective. With a trimmed mean of 5, the equal therapy is preferred over the unequal therapy, which has a trimmed mean of 4. Here, the trimmed mean thus represents the average person better but also ignores that the $20 \%$ largest improvers of the unequal therapy improve massively.

If the research question concerns comparing group averages, then comparing means is most appropriate. Imagine that the research question is which smoking therapy leads to more costs saved for the health care system. To answer this research question, each reduction in the number of cigarettes smoked must be associable with an expected amount of money saved. In this case, choosing the therapy that leads to the biggest average amount of money saved per patient, and thus comparing means, is most appropriate.

An advisable heuristic default approach if all hypotheses are meaningful is to use stochastic equality as the primary hypothesis, as often stochastic equality is what researchers should be most interested in (Cliff, 1996a; Delaney \& Vargha, 2002).

\section{Recurring Example}

The research question concerns a direct comparison of observed scores (do the two groups tend to earn the same?). This indicates that stochastic equality is the most appropriate hypothesis. Examining the example in more detail, investigating whether it is $50 \%$ likely that a random male earns more than a random female is the most appropriate operationalization of whether males and females tend to earn the same.

\section{Test Remaining Meaningful Hypotheses in Secondary Analysis}

Often, the meaningful hypotheses that are not considered most appropriate provide useful complementary information. As was the case for the cigarette example, for which all three hypotheses provided valuable complementary perspectives. Thus, it is advisable to also report on the meaningful hypotheses that were not deemed most appropriate in a secondary analysis.

The obvious drawback of reporting on multiple hypotheses is that by performing multiple hypothesis tests, the probability of falsely rejecting at least one hypothesis (known as familywise error rate) is increased. However, if all null hypotheses are rejected by their tests, the family-wise error rate is not increased (Wilcox, 2017, p. 392). On the other hand, if the most appropriate test is not rejected, it seems unwise not to consider whether any of the other meaningful hypotheses can be rejected. At least, this might indicate what to test as the primary hypothesis when conducting future studies.

\section{Recurring Example}

Since all hypotheses are meaningful, testing equality of means and equality of trimmed means as secondary hypotheses is recommended. For the trimmed means, the default trimming percentage of $20 \%$ is used.

\section{Step Two: Select Tests}

\section{No Test is Optimal}

For each hypothesis, one of the multiple available tests has to be selected. The natural aspiration is to select the optimal test. Unfortunately, for none of the hypotheses, an optimal test exists (Fay \& Proschan, 2010). Consequently, the goal is not to select an optimal test but a test that is the best (on average) of many suboptimal tests. The process of selecting the best test is visualized in Figure 2. 


\section{Figure 2}

Decision tree for step two: Select Tests.

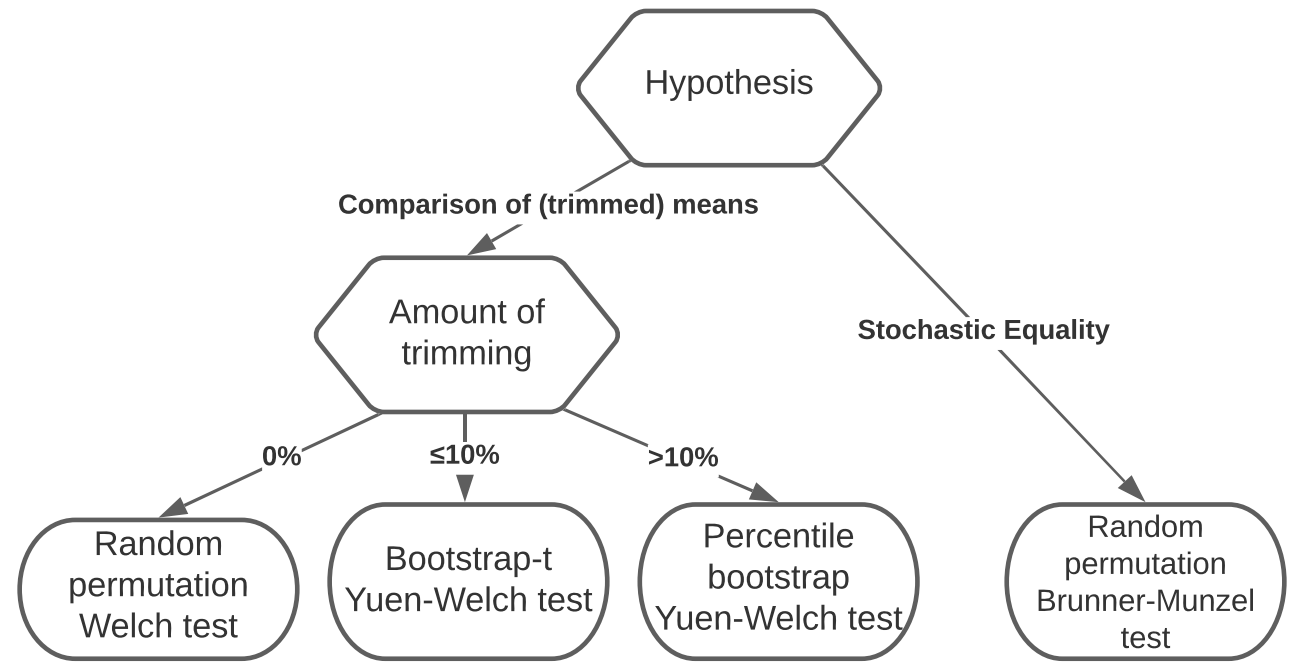

\section{Comparison of Means}

While there is no agreement on which test to use for comparing means, there seems to be an agreement on the test statistic. Delacre et al. (2017), Janssen (1997), Noguchi et al. (2021), Moser and Stevens (1992), Wilcox (2017), Ruxton and Neuhäuser (2019), and Hayes and Cai (2007) all recommend using Welch's test statistic, which allows for unequal variance, over Student's test statistic, or choosing between Welch's and Student's test statistic based on an assessment of the equal variances assumption.

Differences exist regarding the recommended approach for transforming the test statistic to the corresponding $p$ value. Delacre et al. (2017) recommend the commonly used parametric approach, Wilcox (2017) bootstrap resampling, and Janssen (1997), and Noguchi et al. (2021) permutation resampling. Hayes and Cai (2007) compared these three approaches based on type I error rates and found small differences. It is also well known that the differences between the approaches diminish with sample size, as they all behave correctly $^{7}$ in large samples. However, the permutation resampling approach has theoretical advantages. The conditions for which it is guaranteed to have correct type I error rates include more situations than for the other two approaches (Chung \& Romano, 2013). Additionally, Noguchi et al. (2021) found that permutation tests provide better type I error control and higher power than parametric tests in most situations. Janssen and Pauls (2005) found that permutation resampling also provides better type I error rate control and higher power than bootstrapping. Permutation resampling even had the highest power for normally distributed data (Janssen \& Pauls, 2005), arguably the most favorable condition for the parametric approach. Consequently, current evidence favors permutation resampling. The resulting test is known as the random ${ }^{8}$ permutation Welch $t$ test, which is thus also the recommended test for comparing means.

\section{Comparison of Trimmed Means}

The standard test for comparing trimmed means is YuenWelch's test. Modern supposed improvements based on resampling approaches exist but are still understudied. Wilcox (2017), summarizes the current state of knowledge as follows, "Bootstrap methods appear to have an advantage when sample sizes are small (p. 339). ... An educated guess is that the bootstrap-t is preferable if the amount of trimming is less than or equal to $10 \%$ (p.392)", which is thus also the advice for comparing trimmed means given in this paper. Use the bootstrap-t version of the Yuen-Welch test if trimming is less than $10 \%$ if not use the percentile bootstrap version. A special case is $0 \%$ of trimming. In this case, normal means

\footnotetext{
${ }^{7}$ Technically, they are approximately valid and consistent under generous assumptions (Fay \& Proschan, 2010)

${ }^{8}$ Sometimes this test is simply called the permutation Welch $t$ test. I add the qualifier "random" to emphasize that not all permutations are considered but only a random subset. This is in contrast to a vanilla permutation test, which considers all permutations.
} 
are compared, and thus the advice given for comparing means applies instead.

\section{Stochastic Equality}

The standard test for stochastic equality is the WilcoxonMann-Whitney test (Divine et al., 2018). However, the Wilcoxon-Mann-Whitney test has the critical disadvantage that even in large samples it is not guaranteed to perform correctly $^{9}$.

Multiple alternatives addressing this weakness of the Wilcoxon-Mann-Whitney test have been proposed (Brunner et al., 2019; Cliff, 1996b). While there is no consensus yet which of these alternatives should be preferred, the current evidence seems to favor the Brunner-Munzel test weakly. (Karch, 2021). Consequently, I recommend this test for testing stochastic equality.

Equivalently as for Welch's test, the $p$ value of BrunnerMunzel's test can be obtained using multiple approaches. Noguchi et al. (2021) demonstrated that the random permutation approach outperforms the traditional parametric approach both in terms of type I error rates and power, which is thus the approach recommended here.

\section{Step Three: Perform the Selected Tests}

After the hypothesis and corresponding test have been selected, the next step is to gather the data and perform the selected test.

\section{Handle Incorrect Values and Outliers}

Before performing the selected test, an investigation of outliers and incorrect values is crucial. In the context of establishing direction, outliers are defined as unusually large or small values within each group. In contrast, incorrect values are erroneous due to, for example, malfunction of a measurement device or errors during data input. Note that a data point can be incorrect but not an outlier and correct but an outlier.

Outliers and incorrect values should be treated differently (Bakker \& Wicherts, 2014). Incorrect values should be identified and then corrected or deleted. For identifying incorrect values, common outlier detecting methods like box plots can be helpful, as an incorrect value often is an outlier. Note, however, that contrary to common practice, data points should not be removed solely because they are flagged as outliers by a box plot or any other mechanistic outlier detection method. This procedure leads to inflated type I errors (Bakker \& Wicherts, 2014). Valid alternatives to removing outliers are discussed in the next section.

\section{Assess Assumptions}

I limit the discussion to assessing the assumptions for deciding whether the respective test's type I error rate is approximately correct. Assumptions are also crucial for power. However, without knowing the expected effect size, assessing assumptions does not provide any information about power. Instead, a power study is required, which is recommended but beyond the scope of this paper (see Discussion section for pointers).

\section{Incorrect Values}

One implicit assumption of all tests is that there are not too many remaining incorrect values after correction. Especially a comparison of means is highly sensitive to incorrect values (Wilcox, 2016). Consequently, if one cannot exclude the possibility of even small proportions of incorrect values after correction, a comparison of means cannot be performed reliably and should be avoided even if it was deemed the most appropriate hypothesis.

The other two hypotheses are much less susceptible to incorrect data (Bakker \& Wicherts, 2014). Nevertheless, they should also not be tested if it seems that there is a moderate amount of incorrect data, as it might severely impact the type I error rates even in large samples (Wilcox, 2016). In this case, the only remedy is to improve the data quality.

\section{Distributional Assumptions}

First, all tests are level $\alpha$ robust with regard to their distributional assumptions: Under general assumptions (see Janssen, 1997, for the permutation Welch test; Brunner et al., 2019, sec. 3.5.1, for the Brunner-Munzel test; and Lehmann \& Romano, 2005, sec. 15.6, for the Yuen-Welch bootstrap test), which are typically met in psychology, they have the correct type I error rate and a power approaching $100 \%$ in large samples.

Thus instead of checking distributional assumptions in an absolute sense, the question is whether the group sizes are large enough. How large of a group size is needed depends on many factors. In Table 1 , I list the known conditions under which the type I error rate of the tests can be substantially incorrect in small samples. In general, the more severe the problem mentioned, the larger the group sizes needed. Table 1 provides heuristic minimum group sizes for the most used significance threshold of $\alpha=0.05$ beyond which substantially incorrect type I error rates are rare, even if there is a problem. However, as simulation studies can only investigate a subset of all conditions, there is a small probability that researchers will encounter situations for which the required group sizes are larger.

\footnotetext{
${ }^{9}$ Formally, it is not approximately valid (Fay \& Proschan, 2010).
} 
Table 1

Conditions under which problems with type I error rate control can occur in small sample sizes as well as heuristic minimum group sizes beyond which those conditions typically do not cause wrong type I error rates.

\section{Random Permutation Welch Test}

- Problem: Combination of unequal variances, skewed distributions and unequal sample sizes (Pauly et al., 2015).

- Heuristic minimum group sizes: 30 per group, as is commonly recommended (Field, 2017) and confirmed by the results of Delacre et al. (2017).

\section{All Yuen-Welch Tests}

- Problem: Combination of unequal variances, skewed distributions and unequal sample sizes (Yuen, 1974).

- Heuristic minimum group sizes: 30 per group, as type I error rates are typically less affected as for the Welch test (Keselman et al., 2004).

\section{Random Permutation Brunner-Munzel Test}

- Problem: Very unequal group sizes or skewed distributions (Noguchi et al., 2021).

- Heuristic minimum group sizes: 10 per group (Noguchi et al., 2021)

Note. The minimum group sizes apply only to a significance level of $\alpha=0.05$ and were obtained by applying Bradley's (1978) liberal criterion to the studies cited, that is, I determined for each cited simulation study what the minimum group size was beyond which the type I error rate was never larger than 0.075 .

This implies the following strategy for assessing distributional assumptions. First, and most importantly, one should aim at gathering as many observations as possible but at least as many per group as listed in Table 1 . Not only are the tests not robust for smaller samples sizes, but smaller samples sizes also make the study underpowered for all but the biggest effect sizes. If the group sizes are larger than shown in Table 1, one only has to check whether one of the problems mentioned in Table 1 exists in an extreme form. For example, extremely unequal variances and unequal group sizes for the random permutation Welch test.

Multiple strategies can be recommended for deciding whether a particular problem exists in an extreme form, such as relying on theory or investigating based on a pilot study (see, Wells \& Hintze, 2007, for detailed guidance). If it is the only option, it is also admissable to employ the data set used for applying the test, as is traditionally done.

If an extreme problem is found or cannot be ruled out, the advice is to refrain from reporting the respective test's results. The common strategy of transforming the data to alleviate distributional problems cannot be recommended due to its multiple problems (Neuhäuser, 2010).

\section{Recurring example}

With 2077 females and 2070 males, the group sizes are substantially bigger than the heuristic minimum group sizes. The group sizes are also almost equal. Thus, distributional problems would need to be very extreme to cause meaningful type I error rate distortions.

Since no pilot study or other previous data was available, I assessed distributional assumptions primarily using the SLID data set, despite this not being the optimal approach (Wells $\&$ Hintze, 2007). Remember that one problem shared across all two of the three tests is unequal variances. The sample variances were 50.25 for the females and 68.54 for the males. Consequently, the ratio of the two variances was 1.36 , which is obviously not extreme.

The remaining problems are nonnormality / extreme skew. It is well known that income does typically not follow a normal distribution but is positively skewed. I investigated this further based on the SLID data via density plots (shown in Figure 3).

The density plots confirm that the data are positively skewed but not extremely. Thus, considering the large and almost 
Figure 3

Density Plot for the data from the recurring example.

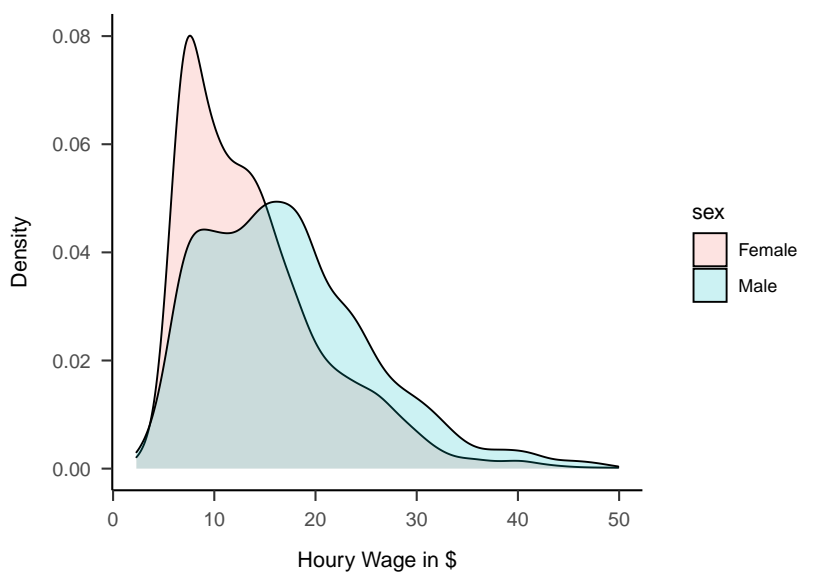

equal group sizes, type I error rates are not meaningfully distorted for any test.

\section{Apply the Test}

After the respective test has been deemed valid, it can be applied. In the supplementary material (https://osf.io/2e6jf/), I demonstrate how to perform all tests in $\mathrm{R}$ based on the recurring example.

\section{Obtain Confidence Intervals}

Additionally to the hypothesis test results, a confidence interval should always be reported to obtain likely values for the magnitude of the difference (Kelley \& Preacher, 2012). All suggested hypotheses tests can be inverted to obtain a corresponding confidence interval for the quantity of interest (Pauly et al., 2016; Wilcox, 2017). As a consequence, the relative robustness of the tests to their distributional assumptions is related to the reliabilities of the corresponding confidence intervals. Consequently, those confidence intervals can be expected to be accurate in most cases. However, especially how to best obtain a confidence interval for stochastic superiority is still an active research topic, and the confidence intervals obtained by inverting the Brunner-Munzel test can be invalid for small samples or when almost all values in one group are bigger than always all values in the other group (Karch, 2021, p. 9). In the supplementary material (https://osf.io/2e6jf/), I demonstrate how to obtain the corresponding confidence interval for each test.

\section{Discussion \& Conclussion}

In this paper, I proposed a new guideline for choosing between hypothesis tests for establishing direction. The central principle is to select the hypothesis that operationalizes the research question most appropriately. Importantly, it is recommended to not only report on the most appropriate hypothesis but also on the remaining meaningful hypotheses in a secondary analysis. Modern, resampling-based hypotheses tests for each hypothesis are recommended.

This novel guideline successfully addresses all the criticism raised against the traditional guideline (see Introduction). By focusing on identifying the meaningful and most appropriate hypotheses independently of the characteristics of the observed data, the observed data do not completely determine the research question and measurement issues are not neglected. Since, additionally, the choice between the BrunnerMunzel test and the $t$ test is primarily based on which hypothesis is most appropriate and not on checking the normality assumption, the preliminary testing paradox is corrected. As modern, resampling-based tests are recommended, modern methods are not ignored. Lastly, since outliers are not removed for trying to reach the assumptions of parametric tests, the outlier removal paradox is remedied.

Throughout the decisions needed for selecting the most appropriate hypothesis, I presented sensible heuristic default choices. Considering all those heuristic default choices together implies that more often than not stochastic equality is most appropriate. In most cases, stochastic equality is the only meaningful hypothesis (see Figure 2). Even if this is not the case, stochastic equality is often the most appropriate hypothesis. Consequently, I recommend that testing for stochastic equality using the Brunner-Munzel test should replace the $t$ test as the default, primary procedure for establishing direction in psychology. Besides being often the most appropriate hypothesis, it is less susceptible to outliers and erroneous data, as well as distributional assumptions (Cliff, 1996b). However, carefully following the suggested guideline should be preferred over just testing for stochastic equality, as for some researchers, it will not be the most appropriate hypothesis.

Beyond choosing the correct hypothesis test, it is also crucial to select the variable that best quantifies the research question. Exemplarily, for the smoking therapy example, an equally valid choice would be to quantify the improvement in the percentage of reduction in cigarettes smoked per day. This might lead to different conclusions than the discussed absolute reduction. Choosing which variable to compare across groups should thus be done with great care before choosing the hypothesis test.

The guideline presented will likely be improved as more knowledge about the relative merits of statistical techniques 
emerge. I will list the topics that I deem the most obvious targets for improvement.

First, while there seems to be a consensus on which test statistic to use for each hypothesis, more research is needed to determine which approach for obtaining the corresponding $p$ value is best under which condition. Specifically, while the advice given is based on up-to-date recommendations and evidence from simulation studies, it is inconsistent. There is no evident reason explaining the differences across tests, in particular, that for the Welch and Brunner-Munzel tests, the random permutation technique is recommended, but for the Yuen-Welch test the bootstrap technique (see Figure 2). Thus, investigating whether more consistent advice, such as always using a random permutation test, would be equally valid is recommended for future work.

Second, more work is needed regarding how to estimate power for the recommended tests. Detailed guidelines are missing for all tests. For the Brunner-Muzel test, no advice exists yet. As a workaround, approaches developed for the WilcoxonMann-Whitney test can be used for now (see, Brunner et al., 2019, sec. 3.8, for an overview), since the Brunner-Munzel test and the Wilcoxon-Mann-Whitney test are similar regarding power (Karch, 2021). For the Yuen-Welch test, Wilcox (2017) provides advice in Section 8.6.5 for the case that pilot data are available. For Welch's test, multiple tools exist (for example, Faul et al., 2009). However, they assume normality, which is often unreasonable in psychology (Micceri, 1989). Estimating power without assuming normality is difficult. One solution could be a simulation study. However, for this to be reliable, the properties of the population distributions, like skewness and kurtosis, need to be known well, which is generally hard to obtain outside of large samples (Wilcox, 2017, sec. 8.6.4).

Third, the space of considered hypotheses and tests should be extended. To make this paper feasible, I focused on the traditional frequentist alternatives of the $t$ test and their variants. Note that those are also the only tests considered by the traditional guideline as well as suggested alternatives (Field, 2017; Rietveld \& van Hout, 2015; Ruxton \& Neuhäuser, 2019). However, many other more specialized hypotheses and methods for establishing direction exist and should also be considered. A prominent alternative over stochastic equality for analyzing ordinal data are latent variable models (Bürkner \& Vuorre, 2019). While they generally make stricter assumptions than stochastic equality (Kampen \& Swyngedouw, 2000), they can have advantages in situations in which these assumptions are plausible. Besides comparing trimmed means, two other prominent robust alternatives to comparing means exist, comparing M-estimators and comparing medians. Beyond this, also Bayesian methods should be considered.

In summary, the proposed guideline for choosing a hypothesis test for establishing direction improves existing strategies, most prominently the widely employed traditional guideline. As such, it should be used in practice. However, it must be continuously extended, reviewed, and updated if needed.

\section{Declarations}

\section{Open Practice}

No materials / data were used for this publication and no experiments have been performed.

\section{Funding}

The author did not receive support from any organization for the submitted work.

\section{Conflicts of interest/Competing interests}

The author has no competing interests to declare that are relevant to the content of this article.

\section{Ethics approval}

Not applicable

\section{Consent to participate}

Not applicable

\section{Consent for publication}

Not applicable

\section{Availability of data and materials}

Not applicable

\section{Code availability}

All code used is available at https://osf.io/qzwrb/.

\section{Authors' contributions}

Remove the maintain integrity of review process 


\section{References}

André, Q. (2021). Outlier exclusion procedures must be blind to the researcher's hypothesis. Journal of Experimental Psychology: General. https://doi.org/10.1037/xge0001069

Bakker, M., \& Wicherts, J. M. (2014). Outlier removal, sum scores, and the inflation of the type I error rate in independent samples t tests: The power of alternatives and recommendations. Psychological Methods, 19(3), 409-427. https: //doi.org/10.1037/met0000014

Bradley, J. V. (1978). Robustness? British Journal of Mathematical and Statistical Psychology, 31(2), 144-152. https://doi.org/10.1111/j.20448317.1978.tb00581.x

Brunner, E., Bathke, A. C., \& Konietschke, F. (2019). Rank and pseudo-rank procedures for independent observations in factorial designs: Using $R$ and $S A S$. Springer International Publishing. https://doi.org/10.1007/978-3-030-02914-2

Brunner, E., \& Munzel, U. (2000). The nonparametric Behrens-Fisher problem: Asymptotic theory and a small-sample approximation. Biometrical Journal, 42(1), 17-25. https: //doi.org/10.1002/(SICI)1521-4036(200001)42: 1\%3C17::AID-BIMJ17\%3E3.0.CO;2-U

Bürkner, P.-C., \& Vuorre, M. (2019). Ordinal regression models in psychology: A tutorial. Advances in Methods and Practices in Psychological Science, 2(1), 77-101. https://doi.org/10. $1177 / 2515245918823199$

Chung, E., \& Romano, J. P. (2013). Exact and asymptotically robust permutation tests. The Annals of Statistics, 41(2), 484-507. https: //doi.org/10.1214/13-AOS1090

Cliff, N. (1996a). Answering ordinal questions with ordinal data using ordinal statistics. Multivariate Behavioral Research, 31(3), 331-350. https://doi.org/10.1207/s15327906mbr3103_4

Cliff, N. (1996b). Ordinal methods for behavioral data analysis. Erlbaum.

Delacre, M., Lakens, D., \& Leys, C. (2017). Why psychologists should by default use Welch's $t$ test Instead of Student's $t$-test. International Review of Social Psychology, 30(1), 92. https: //doi.org/10.5334/irsp.82

Delaney, H. D., \& Vargha, A. (2002). Comparing several robust tests of stochastic equality with ordinally scaled variables and small to moderate sized samples. Psychological Methods, 7(4), 485-503. https://doi.org/10.1037/1082989X.7.4.485

Divine, G. W., Norton, H. J., Barón, A. E., \& Juarez-
Colunga, E. (2018). The Wilcoxon-MannWhitney procedure fails as a test of medians. The American Statistician, 72(3), 278-286. https: //doi.org/10.1080/00031305.2017.1305291

Faul, F., Erdfelder, E., Buchner, A., \& Lang, A.G. (2009). Statistical power analyses using $\mathrm{G}^{*}$ Power 3.1: Tests for correlation and regression analyses. Behavior Research Methods, 41(4), 1149-1160. https://doi.org/10.3758/BRM. 41.4.1149

Fay, M. P., Brittain, E. H., Shih, J. H., Follmann, D. A., \& Gabriel, E. E. (2018). Causal estimands and confidence intervals associated with Wilcoxon-Mann-Whitney tests in randomized experiments. Statistics in Medicine, 37(20), 29232937. https://doi.org/10.1002/sim.7799

Fay, M. P., \& Proschan, M. A. (2010). WilcoxonMann-Whitney or t-test? On assumptions for hypothesis tests and multiple interpretations of decision rules. Statistics Surveys, 4, 1-39. https: //doi.org/10.1214/09-SS051

Field, A. (2017). Discovering statistics using IBM SPSS statistics (5th ed.). Sage.

Fox, J. (2016). Applied regression analysis and generalized linear models (3rd ed.). Sage.

Fox, J., Weisberg, S., \& Price, B. (2020). carData: Companion to applied regression data sets [Manual]. https://CRAN.R-project.org/ package $=$ carData

Good, P. (2005). Permutation, parametric and bootstrap tests of hypotheses (3. ed). Springer.

Hayes, A. F., \& Cai, L. (2007). Further evaluating the conditional decision rule for comparing two independent means. British Journal of Mathematical and Statistical Psychology, 60(2), 217244. https://doi.org/10.1348/000711005X62576

Janssen, A. (1997). Studentized permutation tests for non-i.i.d. Hypotheses and the generalized Behrens-Fisher problem. Statistics \& Probability Letters, 36(1), 9-21. https://doi.org/10.1016/ S0167-7152(97)00043-6

Janssen, A., \& Pauls, T. (2005). A Monte Carlo comparison of studentized bootstrap and permutation tests for heteroscedastic two-sample problems. Computational Statistics, 20(3), 369-383. https://doi.org/10.1007/BF02741303

Kampen, J., \& Swyngedouw, M. (2000). The ordinal controversy revisited. Quality \& Quantity, 34, 87-102.

Karch, J. D. (2021). Psychologists should use Brunner-Munzel's instead of Mann-Whitney's $\mathrm{U}$ test as the default nonparametric procedure. Advances in Methods and Practices in Psycho- 
logical Science, 4(2). https://doi.org/10.1177/ 2515245921999602

Kelley, K., \& Preacher, K. J. (2012). On effect size. Psychological Methods, 17(2), 137-152. https://doi.org/10.1037/a0028086

Keselman, H. J., Othman, A. R., Wilcox, R. R., \& Fradette, K. (2004). The New and Improved Two-Sample t Test. Psychological Science, 15(1), 47-51. https://www.jstor.org/stable/ 40063824

Lehmann, E. L., \& Romano, J. P. (2005). Testing statistical hypotheses (3rd ed.). Springer.

Lord, F. M. (1953). On the statistical treatment of football numbers. American Psychologist, 8(12), 750-751. https://doi.org/10.1037/h0063675

Micceri, T. (1989). The unicorn, the normal curve, and other improbable creatures. Psychological Bulletin, 105(1), 156-166. https://doi.org/10. 1037/0033-2909.105.1.156

Moser, B. K., \& Stevens, G. R. (1992). Homogeneity of Variance in the Two-Sample Means Test. The American Statistician, 46(1), 19-21. https://doi.org/10.2307/2684403

Neubert, K., \& Brunner, E. (2007). A studentized permutation test for the non-parametric Behrens-Fisher problem. Computational Statistics \& Data Analysis, 51(10), 5192-5204. https: //doi.org/10.1016/j.csda.2006.05.024

Neuhäuser, M. (2010). A nonparametric two-sample comparison for skewed data with unequal variances. Journal of Clinical Epidemiology, 63(6), 691-693. https://doi.org/10.1016/j.jclinepi.2009. 08.026

Noguchi, K., Konietschke, F., Marmolejo-Ramos, F., \& Pauly, M. (2021). Permutation tests are robust and powerful at $0.5 \%$ and $5 \%$ significance levels. Behavior Research Methods. https: //doi.org/10.3758/s13428-021-01595-5

Pauly, M., Asendorf, T., \& Konietschke, F. (2016). Permutation-based inference for the AUC: A unified approach for continuous and discontinuous data. Biometrical Journal, 58(6), 1319-1337. https://doi.org/10.1002/bimj.201500105

Pauly, M., Brunner, E., \& Konietschke, F. (2015). Asymptotic permutation tests in general factorial designs. Journal of the Royal Statistical Society: Series B (Statistical Methodology), 77(2), 461473. https://doi.org/10.1111/rssb.12073

Rasch, D., Kubinger, K. D., \& Moder, K. (2011). The two-sample t test: Pre-testing its assumptions does not pay off. Statistical Papers, 52(1), 219-231. https://doi.org/10.1007/s00362-0090224-x
Rietveld, T., \& van Hout, R. (2015). The t test and beyond: Recommendations for testing the central tendencies of two independent samples in research on speech, language and hearing pathology. Journal of Communication Disorders, 58, 158-168. https://doi.org/10.1016/j.jcomdis.2015. 08.002

Ruxton, G. D. (2006). The unequal variance t-test is an underused alternative to Student's t-test and the Mann-Whitney U test. Behavioral Ecology, 17(4), 688-690. https://doi.org/10.1093/beheco/ $\operatorname{ark} 016$

Ruxton, G. D., \& Neuhäuser, M. (2019). Striving for simple but effective advice for comparing the central tendency of two populations. Journal of Modern Applied Statistical Methods, 17(2), eP2567. https://doi.org/10.22237/jmasm/ 1551908612

Schlag, K. H. (2015). Who gives direction to statistical testing? Best practice meets mathematically correct tests. SSRN Electronic Journal. https://doi.org/10.2139/ssrn.2660977

Stevens, S. S. (1946). On the Theory of Scales of Measurement. Science, 103(2684), 677-680. https://doi.org/10.1126/science.103.2684.677

Umbach, N., Naumann, K., Brandt, H., \& Kelava, A. (2017). Fitting Nonlinear Structural Equation Models in R with Package nlsem. Journal of Statistical Software, 77(1, 1), 1-20. https: //doi.org/10.18637/jss.v077.i07

Velleman, P. F., \& Wilkinson, L. (1993). Nominal, ordinal, interval, and ratio typologies are misleading. The American Statistician, 47(1), 65-72. https://doi.org/10.1080/00031305.1993. 10475938

Wells, C. S., \& Hintze, J. M. (2007). Dealing with assumptions underlying statistical tests. Psychology in the Schools, 44(5), 495-502. https: //doi.org/10.1002/pits.20241

Wilcox, R. R. (2016). Introduction to robust estimation and hypothesis testing (4th ed.). Elsevier.

Wilcox, R. R. (2017). Modern Statistics for the Social and Behavioral Sciences. CRC Press. 